\title{
Predictors of Weight Bias in Exercise Science Students and Fitness Professionals: A Scoping Review
}

\author{
Lara Zaroubi, Tiffany Samaan, and Angela S. Alberga \\ Department of Health, Kinesiology \& Applied Physiology, Concordia University, Montreal, QC, Canada \\ Correspondence should be addressed to Angela S. Alberga; angela.alberga@concordia.ca
}

Received 28 January 2021; Accepted 10 June 2021; Published 6 July 2021

Academic Editor: Nunzio Velotti

Copyright (C) 2021 Lara Zaroubi et al. This is an open access article distributed under the Creative Commons Attribution License, which permits unrestricted use, distribution, and reproduction in any medium, provided the original work is properly cited.

Background. Although previous studies have reported weight bias among students and professionals in exercise science, physical education, kinesiology, and fitness instruction, predictors of weight bias in these professions have not been extensively reviewed. Aim. The purpose of this scoping review was to explore the available literature on predictors of weight bias in exercise science students and fitness professionals to identify key concepts and research gaps. Methods. PubMed and ERIC were searched from January 1990 to May 2019. Eighteen studies were included in this review. A thematic analysis was conducted. Findings. Six main themes were drawn from these studies including beliefs in the personal controllability of weight; sex differences; enrollment in a health sciences-related program; psychosocial and personal factors; knowledge of obesity; lack of personal history, family, or friend with obesity. Our scoping review highlighted diverse predictors of weight bias among exercise science students and professionals that warrant further study and intervention.

\section{Introduction}

Weight bias is defined as negative beliefs and attitudes toward people living with overweight or obesity [1]. Beliefs and opinions that occur in a conscious and expressive manner are defined as explicit forms of weight bias [2]. Previous studies have documented the existence of explicit weight bias in various settings such as in healthcare among trainees (i.e., nursing, dietetic, and medical students) [2], education (i.e., schoolteachers) [3], and medicine and public health [4-8]. Healthcare providers often associate people with obesity with negative labels and stereotypes such as "lazy", "weak", "lack willpower", "unattractive", or "unintelligent" $[9,10]$. In fact, patients with obesity have reported low trust, poor communication, lack of training, and disrespectful treatment from their healthcare providers [11]. Experiencing weight bias in healthcare settings is particularly harmful because it can negatively affect patient engagement and utilization of healthcare services [11]. Future health professionals' biases are problematic as they may deter both clients and patients from adopting healthy lifestyle choices $[12,13]$. For example, individuals with obesity experiencing explicit weight bias can experience physical and emotional tribulations including stress, anxiety, depression, avoidance or lower motivation for exercise, and disordered eating [14] and may not be receiving appropriate care for their health conditions [15].

While there is a lack of clearly defined approaches to reduce weight bias among healthcare professionals, a systematic review of weight bias reduction interventions identified preprofessional educational training in healthcare programs as one potential target [16]. Since many healthcare professionals and health educators working with adults and children with obesity often have educational training backgrounds in physical education, health, and kinesiology, it would be important to understand predictors of weight bias among students and professionals in these fields. Numerous studies have reported weight bias among students in physical education [17], kinesiology, and exercise science programs $[18,19]$ and professionals in these fields (e.g., physical education teachers, fitness instructors) $[20,21]$. Weight bias has been observed among physical education teachers whereby they have expressed lower expectations in performance and abilities of students with obesity compared 
to their normal weight peers [20]. Students enrolled in physical education programs who have not addressed their own weight-biased attitudes throughout their training program may express these biases as future physical education teachers toward their own students with obesity [20]. In fact, physical educators' weight bias, lower expectations, and experiences of weight teasing of children with obesity may lead to poorer self-esteem, body image issues, lower physical activity performance and lower motivation if students experience differential treatment because of their body weight [22-24]. There is evidence that suggests physical education students display greater weight bias toward the third year of their program compared to their first year and display higher weight bias compared to other health science students [17]. This further highlights the importance of understanding factors associated with weight bias in the early formative years to avoid the propagation of weight bias in professional practice and its potential to negatively affect the treatment and quality of healthcare of individuals with obesity.

While it is clear that weight bias is pervasive in students and professionals in the field of exercise science, we need a better understanding of the factors that predict weight bias in this field to avoid the propagation of weight bias and its negative consequences on patient adherence, health behaviors, and clinical outcomes. The purpose of this scoping review was to synthesize all available literature pertaining to the predictors of weight bias in students and professionals in exercise sciences. For the purpose of this paper, students in exercise science refer to students enrolled in undergraduate or graduate programs in exercise science, physical education, and kinesiology and professionals in exercise science include physical educators, fitness instructors, exercise physiologists, and exercise specialists. We used a scoping review methodology due to the diverse body of literature on this topic and large range of study designs and methodologies before considering undertaking a systematic review. This scoping review further aimed to identify knowledge gaps and future research directions in the field of weight bias among exercise and fitness trainees and professionals.

\section{Methods}

We conducted a scoping review using the five-stage methodology outlined by Arksey and O'Malley [25]. With this method, all evidence and sources pertaining to our research question were gathered and summarized into overarching themes [25]. As such, scoping reviews are guided by a requirement to identify all relevant literature regardless of the heterogeneity of the body of literature, design, or quality [26]. This methodological approach is effective in presenting a broad overview of the literature on our research topic and is an effective way to identify research gaps [25]. The Preferred Reporting Items for Systematic reviews and Meta-Analyses extension for Scoping Reviews (PRISMA-ScR) flow diagram (see Figure 1) and checklist (Table 1) [27] were used to guide the reporting and show the steps taken in the article selection process of this review (see Appendix).
2.1. Literature Search. A literature search was designed and conducted in consultation with a health sciences librarian. PubMed and ERIC databases were searched on December 14, 2017, and again on May 8, 2019, using combinations of keywords and subject terms for weight, bias, health science students, and fitness professionals. Results were limited to articles published after January 1, 1990, and written in English or French. The complete search strategy for both databases can be found in the Appendix. Other articles $(n=2)$ were retrieved from the reference lists of pertinent studies that were identified in the personal libraries of the researchers.

2.2. Study Selection. Research studies that sought to understand the predictors and causes of weight bias in exercise science students and professionals were included in this scoping review. Only articles that included students and professionals in the fields of exercise science, physical education, kinesiology, physical therapy, fitness instruction, and exercise physiology were eligible for inclusion. Among selected articles, only those measuring the predictors or potential causes of weight bias in exercise science students and professionals were included for analysis. Studies that assessed weight bias in practicing health professionals including physicians, nurses, doctors, dietitians, psychologists, and social workers were not included in this review.

2.3. Data Charting. Reviewers (L.Z and T.S.) charted the characteristics of included studies in a table outlining title, authors, date of publication, country, study purpose, participant characteristics, methodology, and main findings. All authors verified the charted data for accuracy and the data are presented in Table 2.

\section{Results}

The literature search conducted on May 8, 2019, resulted in 1310 unique articles (after 9 duplicate articles were removed). An additional two articles were identified from the reference lists from the researchers' personal libraries resulting in a total of 1312 articles. These 1312 articles were screened and assessed for eligibility based on the inclusion criteria. Of the 41 articles that were screened as potentially relevant, 18 studies met the eligibility criteria and were included in the scoping review (Figure 1).

3.1. Characteristics of Included Studies. Table 2 shows the characteristics of included studies. More than half of the studies included were conducted in the USA $(61.11 \%, n=11)$ and used a combination of implicit and explicit measures to assess weight bias $(61.11 \%, n=11)$. The rest of the studies measured weight bias through explicit measures only $(27.77 \%, n=5)$ or through other measures $(11.11 \%, n=2)$ such as Q-methodology and one-on-one interviews to explore personal constructs of body shape and weight.

The majority of the studies $(66.67 \%, n=12)$ sampled undergraduate students enrolled in exercise science $(n=3)$, 


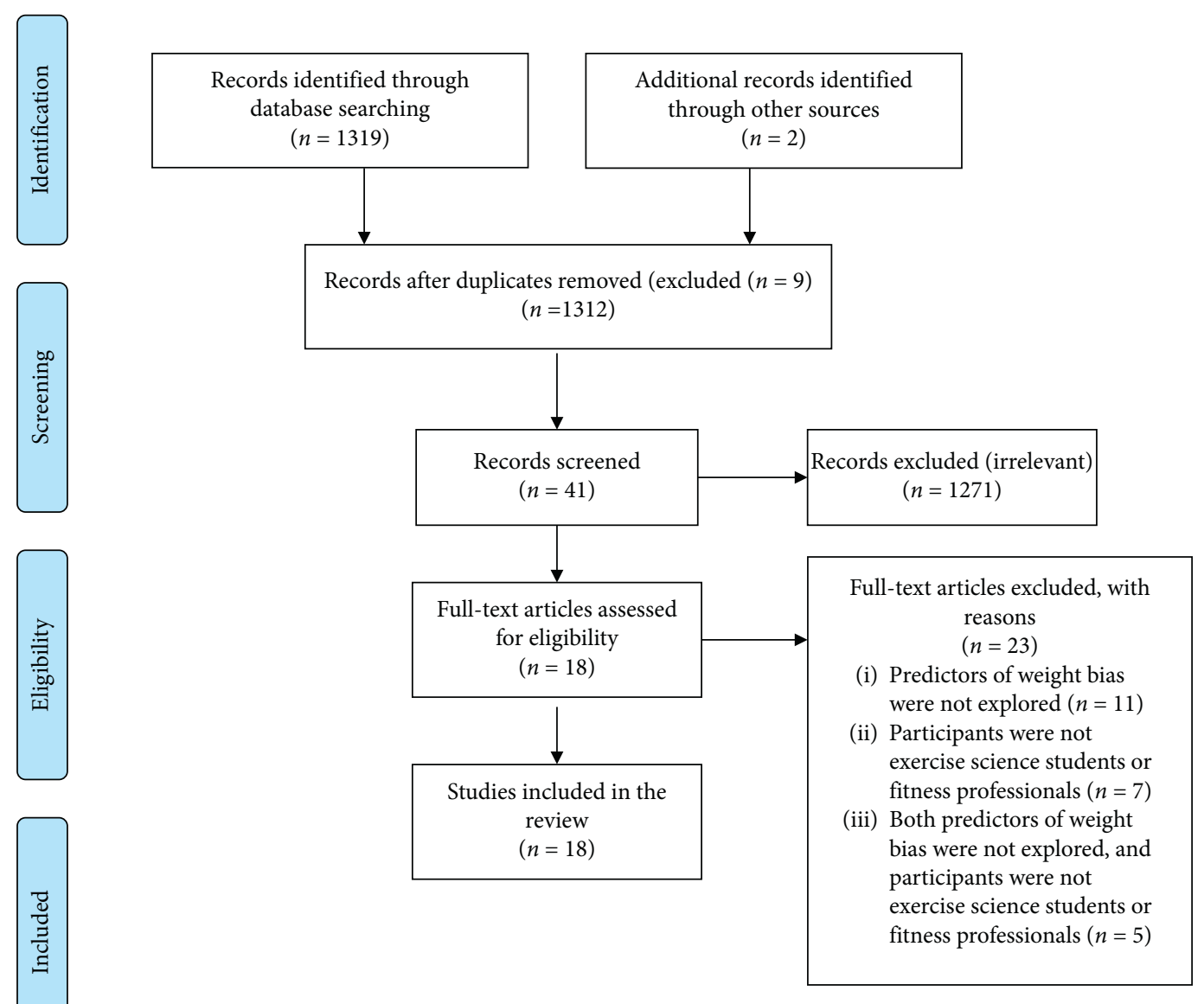

FIGURE 1: PRISMA-ScR flowchart illustrating the article selection process for the literature search.

kinesiology $(n=3)$, physiology $(n=1)$, kinesiology, health promotion, and recreation $(n=1)$, health sciences $(n=1)$, and health and physical education training programs $(n=3)$. Four of the 18 studies $(22.22 \%, n=4)$ sampled fitness professionals only which included personal trainers and fitness instructors $(n=1)$, health educators $(n=1)$, fitness center employees $(n=1)$, and physical education professors $(n=1)$. Finally, one study $(5.55 \%, n=1)$ sampled both exercise science students and fitness professionals, e.g., physical education and exercise science students and athletes.

3.2. Themes. While conducting the thematic analysis according to Arksey and O'Malley [25], the researchers identified and grouped similar themes from each study' findings. Next, similar group themes were further synthesized into overarching themes. A total of six themes emerged to explain the predictors of weight bias in students and professionals in exercise science: beliefs in the controllability of weight; sex or gender differences; enrollment in a health sciences degree or program; psychosocial and personal factors; knowledge of obesity; lack of personal history, family, or friend with obesity. studies $[18,21,23,33,36-39]$ showed that exercise science students and professionals generally believe that weight is personally controllable. Common statements included "everyone has control over their weight" [39]; "eating right and exercising puts you on the right path for a long healthy life" [36]; and "physical activity is very important in the treatment of obesity" [33].

Exercise science students and professionals endorsing strong beliefs in weight controllability tended to explicitly associate people with obesity with "bad" attributes [21], "lazy" stereotypes and held higher explicit weight bias on social/character disparagement and weight control/blame attributes [18].

3.4. Sex Differences. Five studies reported sex or gender differences in the perception of weight toward individuals with obesity [18, 21, 29, 34, 35]. Two out of five studies found that men displayed higher weight bias attitudes than women $[34,35]$. Indeed, according to Langdon et al., Exercise Science/Health students (ESHS) who were male tended to hold stronger explicit weight bias beliefs on weight control/ blame, social/character disparagement, and physical/romantic subscales than ESHS who were female [34]. Alternatively, two studies found that women had stronger implicit weight bias toward individuals with obesity $[18,21]$ and were more likely to implicitly describe them as "bad" 
TABle 1: Preferred Reporting Items for Systematic reviews and Meta-Analyses extension for Scoping Reviews (PRISMA-ScR) checklist.

\begin{tabular}{lcccc}
\hline Section & Item & PRISMA-ScR checklist ITEM & $\begin{array}{c}\text { Reported on } \\
\text { PAGE \# }\end{array}$ \\
$\begin{array}{lccc}\text { Title } \\
\text { Title }\end{array}$ & 1 & Identify the report as a scoping review. & Page 1 \\
\hline
\end{tabular}

Abstract

Structured summary

Provide a structured summary that includes (as applicable): background,

Introduction

Rationale

Methods

Protocol and registration

Eligibility criteria

Information sources*

Search

Selection of sources of evidence $\dagger$

Data charting process ${ }^{\ddagger}$

Data items

Critical appraisal of individual sources of evidence§

Synthesis of results

Results

Selection of sources of evidence

Characteristics of sources of evidence

Critical appraisal within sources of evidence

Results of individual sources of evidence

Synthesis of results

Discussion

Summary of evidence consider the relevance to key groups.

Limitations

Conclusions used in any data synthesis (if appropriate).

Give numbers of sources of evidence screened, assessed for eligibility, and
2 objectives, eligibility criteria, sources of evidence, charting methods, results, and conclusions that relate to the review questions and objectives.

Summarize the main results (including an overview of concepts, themes, and 19 types of evidence available), link to the review questions and objectives, and

Describe the rationale for the review in the context of what is already known.

3 Explain why the review questions/objectives lend themselves to a scoping review approach.

Provide an explicit statement of the questions and objectives being addressed with reference to their key elements (e.g., population or participants, concepts, and context) or other relevant key elements used to conceptualize the review questions and/or objectives.

Indicate whether a review protocol exists; state if and where it can be accessed 5 (e.g., a Web address); and if available, provide registration information, including the registration number.

Specify characteristics of the sources of evidence used as eligibility criteria 6 (e.g., years considered, language, and publication status), and provide a rationale.

Describe all information sources in the search (e.g., databases with dates of 7 coverage and contact with authors to identify additional sources), as well as the date the most recent search was executed.

Present the full electronic search strategy for at least 1 database, including any limits used, such that it could be repeated.

State the process for selecting sources of evidence (i.e., screening and eligibility) included in the scoping review.
escribe the methods of charting data from the included sources of evidence

Page 2 (e.g., calibrated forms or forms that have been tested by the team before their use, and whether data charting was done independently or in duplicate) and any processes for obtaining and confirming data from investigators.

1 List and define all variables for which data were sought and any assumptions and simplifications made.

If done, provide a rationale for conducting a critical appraisal of included 2 sources of evidence; describe the methods used and how this information was

Describe the methods of handling and summarizing the data that were charted.

Pages 3-5

Pages 4-6 Page 5

Pages 5-6

Pages 5

Pages 34-36

Pages 5-6

Pages 6, 20-33

Pages $20-33$

N/A

Pages 6, 20-33 14 included in the review, with reasons for exclusions at each stage, ideally using a flow diagram.

15 For each source of evidence, present characteristics for which data were charted and provide the citations.

16 If done, present data on critical appraisal of included sources of evidence (see item 12).

Page 19

Pages 20-33

N/A

Pages 20-33

Pages 20-33

Summarize and/or present the charting results as they relate to the review questions and objectives.

Pages 6-11

Discuss the limitations of the scoping review process.

Provide a general interpretation of the results with respect to the review questions and objectives, as well as potential implications and/or next steps.
Pages 14-15

Page 15 
TABLE 1: Continued.

\begin{tabular}{|c|c|c|c|}
\hline Section & Item & PRISMA-ScR checklist ITEM & $\begin{array}{c}\text { Reported on } \\
\text { PAGE \# }\end{array}$ \\
\hline \multicolumn{4}{|l|}{ Funding } \\
\hline Funding & 22 & $\begin{array}{l}\text { Describe sources of funding for the included sources of evidence, as well as } \\
\text { sources of funding for the scoping review. Describe the role of the funders of } \\
\text { the scoping review. }\end{array}$ & Page 15 \\
\hline \multicolumn{4}{|c|}{$\begin{array}{l}\mathrm{JBI}=\text { Joanna Briggs Institute; PRISMA-ScR = Preferred Reporting Items for Systematic reviews and Meta-Analyses extension for Scoping Reviews. *Where } \\
\text { sources of evidence (see second footnote) are compiled from, such as bibliographic databases, social media platforms, and Web sites. }{ }^{+} \mathrm{A} \text { more inclusive/ } \\
\text { heterogeneous term used to account for the different types of evidence or data sources (e.g., quantitative and/or qualitative research, expert opinion, and } \\
\text { policy documents) that may be eligible in a scoping review as opposed to only studies. This is not to be confused with information sources (see first footnote). } \\
\text { "The frameworks by Arksey and O'Malley (6) and Levac and colleagues (7) and the JBI guidance }(4,5) \text { refer to the process of data extraction in a scoping } \\
\text { review as data charting. 'The process of systematically examining research evidence to assess its validity, results, and relevance before using it to inform a } \\
\text { decision. This term is used for items } 12 \text { and } 19 \text { instead of "risk of bias" (which is more applicable to systematic reviews of interventions) to include and } \\
\text { acknowledge the various sources of evidence that may be used in a scoping review (e.g., quantitative and/or qualitative research, expert opinion, and policy } \\
\text { document). }\end{array}$} \\
\hline
\end{tabular}

$[18,21]$ and "lazy" [21]. Two studies found no statistically significant differences in antifat bias scores between males and females [28, 29]. Although there is a relationship between sex and gender differences and weight bias, the studies have shown opposing findings in predicting the direction of the relationship with sex and gender.

\subsection{Enrollment in a Health Sciences Degree or Program.} Four studies compared weight bias in students majoring in health and exercise science to students enrolled in nonhealth disciplines, e.g., business, psychology, and other nonhealth majors $[10,17,32]$. Three studies showed that physical education and kinesiology students have higher weight bias compared to students enrolled in nonhealth degree programs [23]. O’Brien et al. showed that physical education students displayed higher levels of implicit and explicit weight bias as compared to psychology students [17]. Lynagh et al. found that enrollment in the health and physical education (HPE) specialist degree was a significant predictor of implicit weight bias and that HPE students held higher levels of weight bias compared to students in the nonspecialist teaching degree program [23]. Greenleaf et al. showed that students enrolled in Kinesiology, Health Promotion and Recreation (KHPR) had higher levels of explicit weight bias and endorsed stereotypes for children with obesity such as "likely to be teased", "bad to be", and "lazy" compared to non-KHPR majors [32]. However, one study by Robinson et al. found similar levels of both implicit and explicit weight bias in health science students enrolled in medicine, medical science, nursing/midwifery, pharmacy, dietetics, public health, exercise science, physiotherapy, etc., compared to nonhealth majors in business programs [10].

Interestingly, differences in levels of implicit weight bias were also found between year one and year three physical education students whereby third year students displayed higher levels of weight bias than first year physical education students $[17,19]$.

Enrollment in a health and exercise science program is a potential predictor of weight bias, although more research is needed to determine if weight bias increases in students enrolled in kinesiology or exercise science throughout the duration of their undergraduate degrees.

3.6. Psychosocial and Personal Factors. Six studies associated psychosocial factors, professional philosophies, and perceptions of self with weight bias in exercise science students and professionals [17, 29, 31, 33-35]. Psychosocial factors such as having ego-oriented goals and a tendency to internalize the athletic body ideal were measured in exercise science students. Students with ego-oriented goals "may avoid challenging tasks and feel discouraged when their performance is perceived as inferior to others" [34]. In this study, exercise science students were also likely to exhibit high internalization of the athletic body ideal and were "particularly susceptible to media messaging that idealizes the athletic body, portrayed as competent, competitive, and healthy" [34]. High internalization of the athletic body type ideal among exercise science students was found to be a predictor of fat phobia and weight control blame [34].

Social dominance orientation was cited as a predictor for explicit and implicit measures of weight bias whereby physical education students "see their own group as superior to and dominant over other relevant groups" [17]. Physical education students with high social dominance orientation displayed weight bias on explicit measures such as "dislike", "fear of fat", and "lack of willpower" and the implicit measures "bad" and "lazy" [17].

In fitness professionals, three studies have associated professional philosophies and perception of self with weight bias $[17,29,35]$. One study looked at different professional philosophies such as "behavior change", "cognitive-based", "decision-making", "freeing/functioning", and "social change" [29]. Among all professional philosophy measures, it was found that the "behavior change" education philosophy (i.e., emphasizing behavior modification as key in managing obesity) was associated with higher explicit weight bias in health educators [29]. In another study by MartinezLopez et al., 2010, self-efficacy expectations were measured to assess weight bias in physical education trainees toward youth with obesity. Self-efficacy in physical education trainees was defined as "the perceptions about their own 


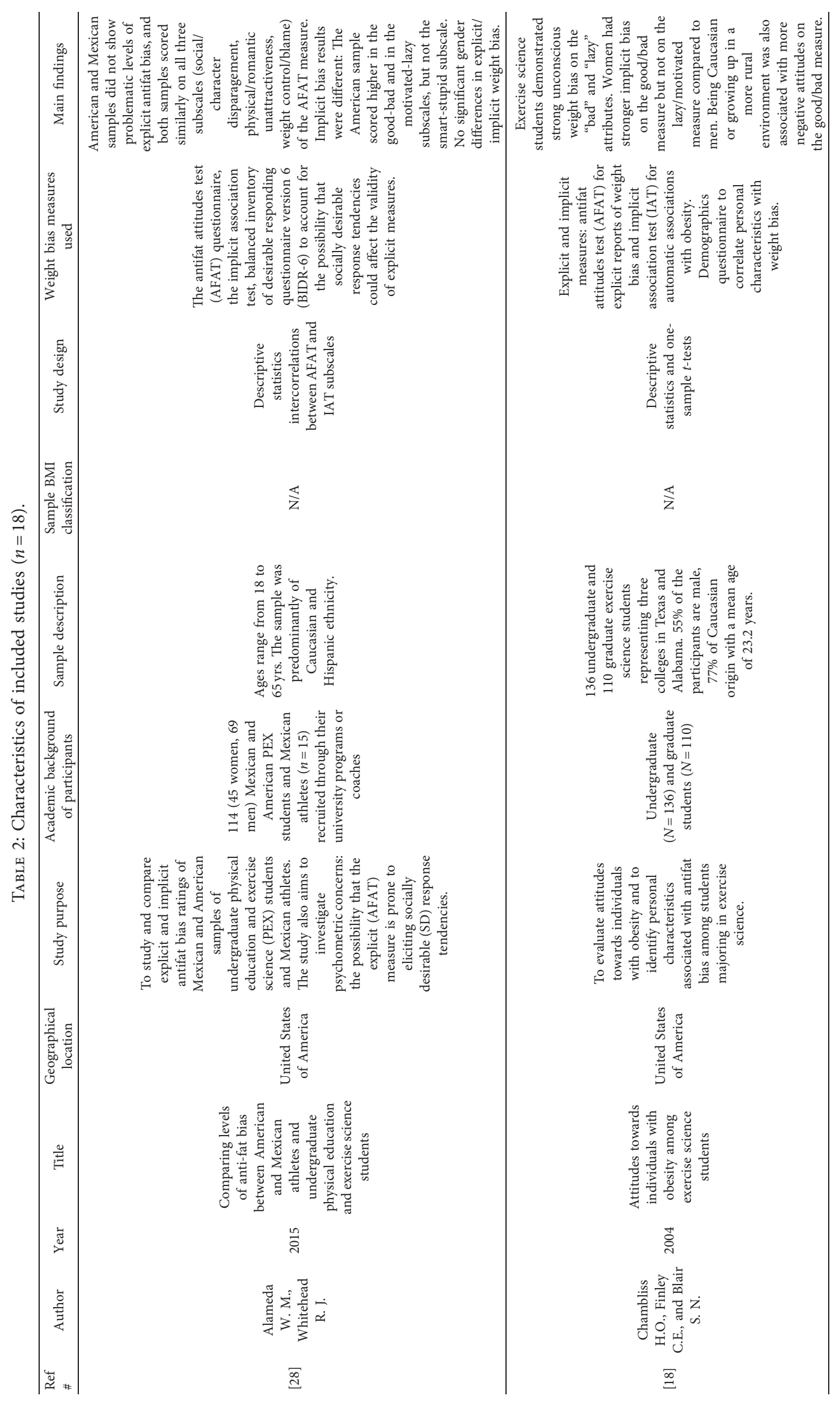




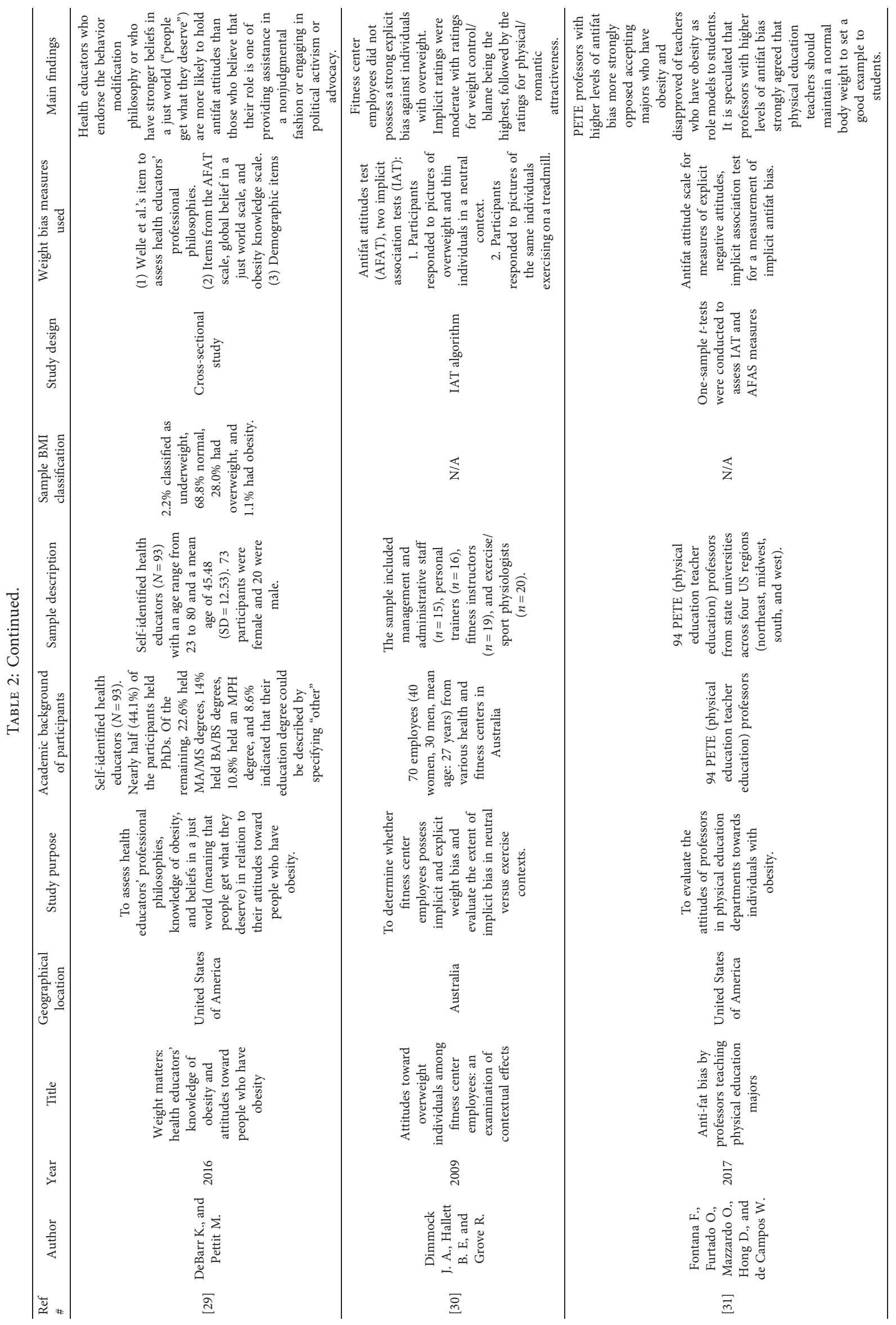




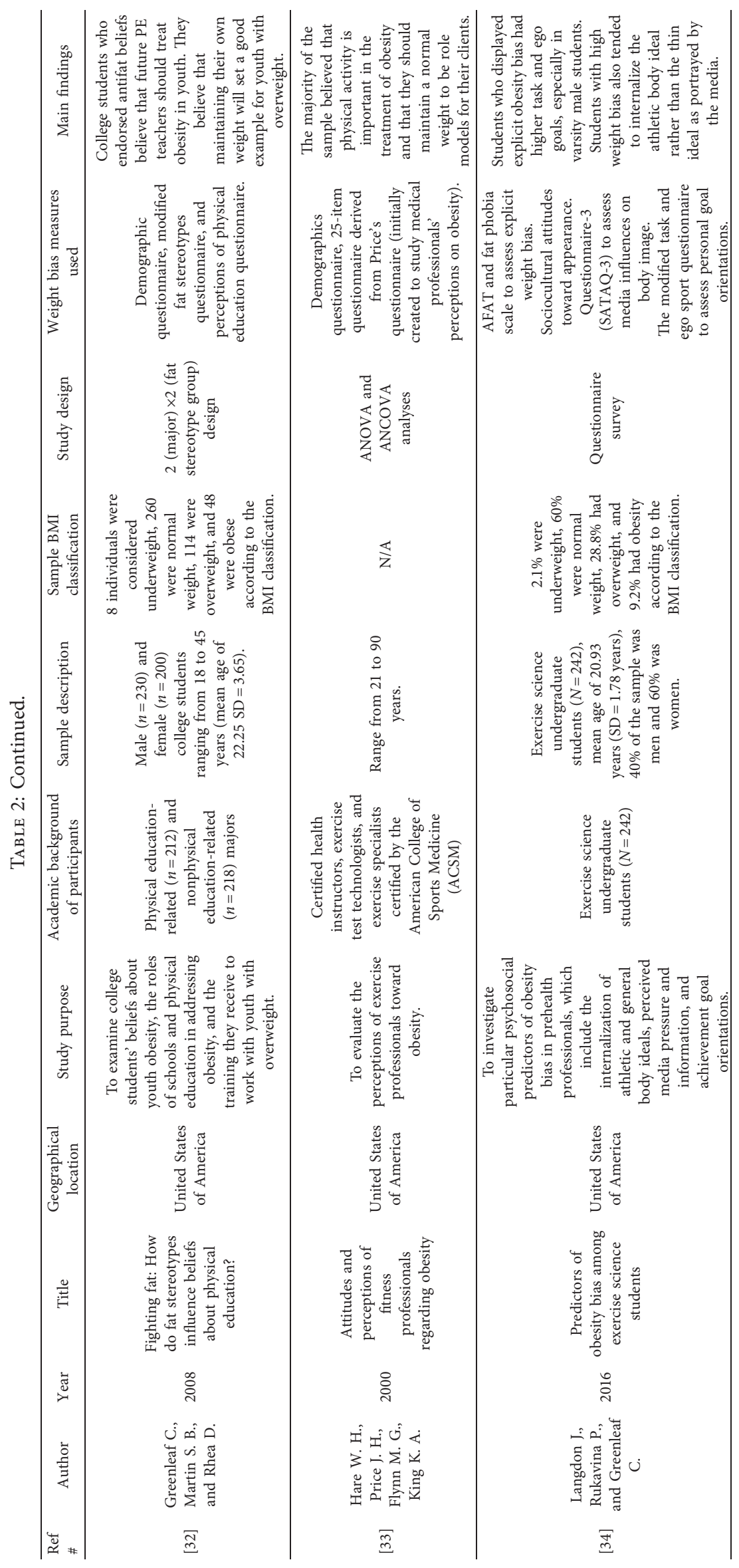




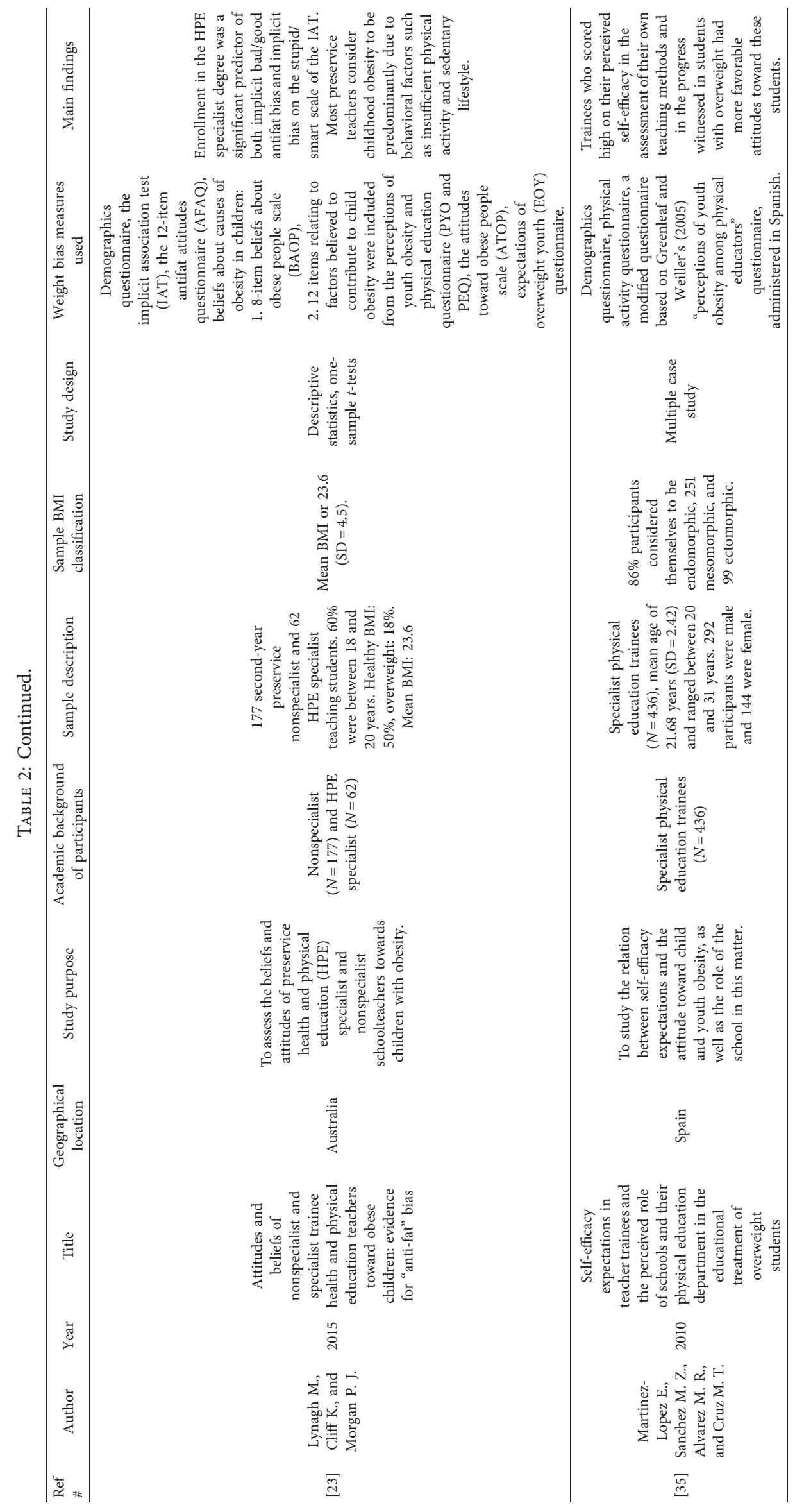




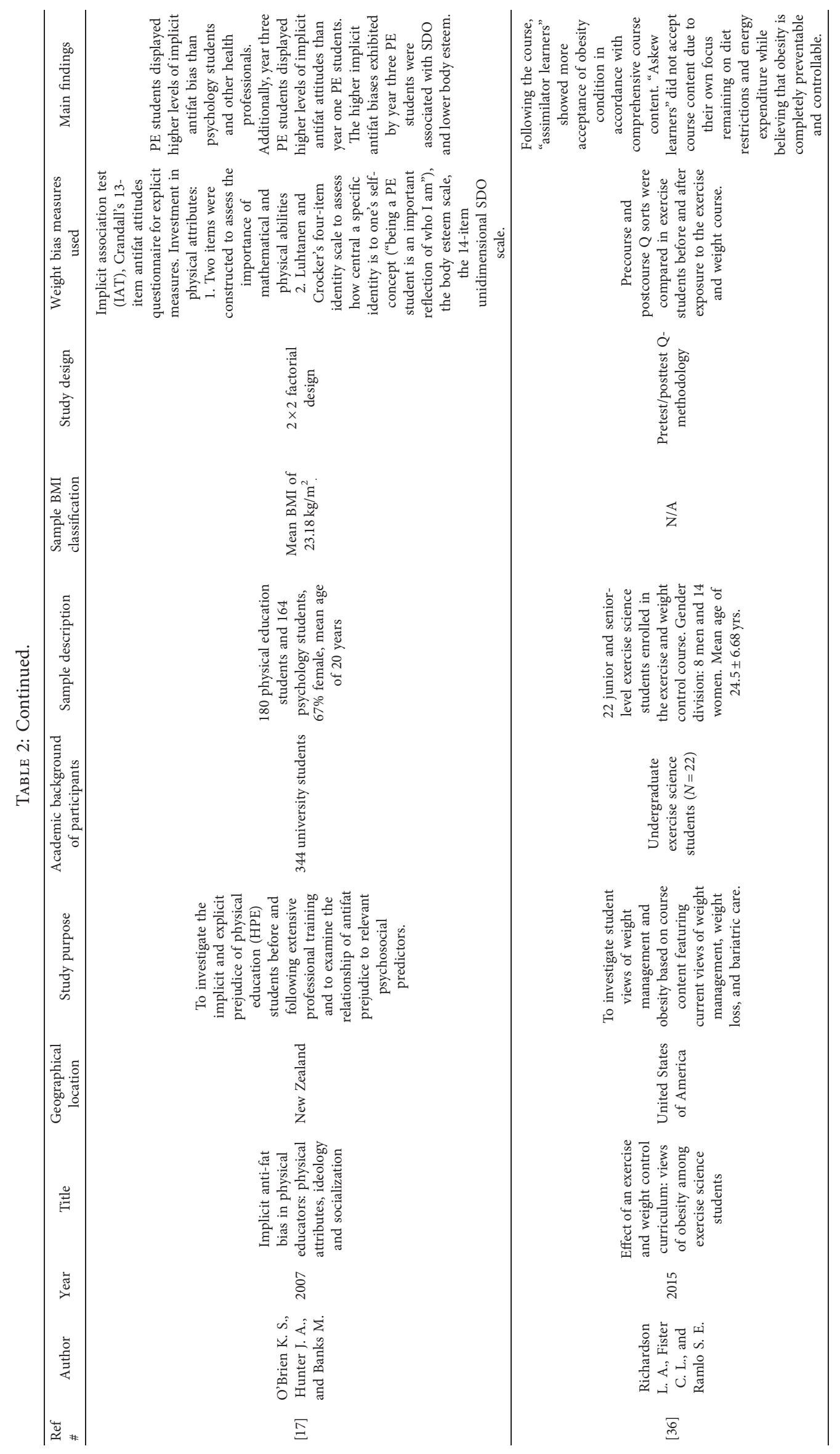




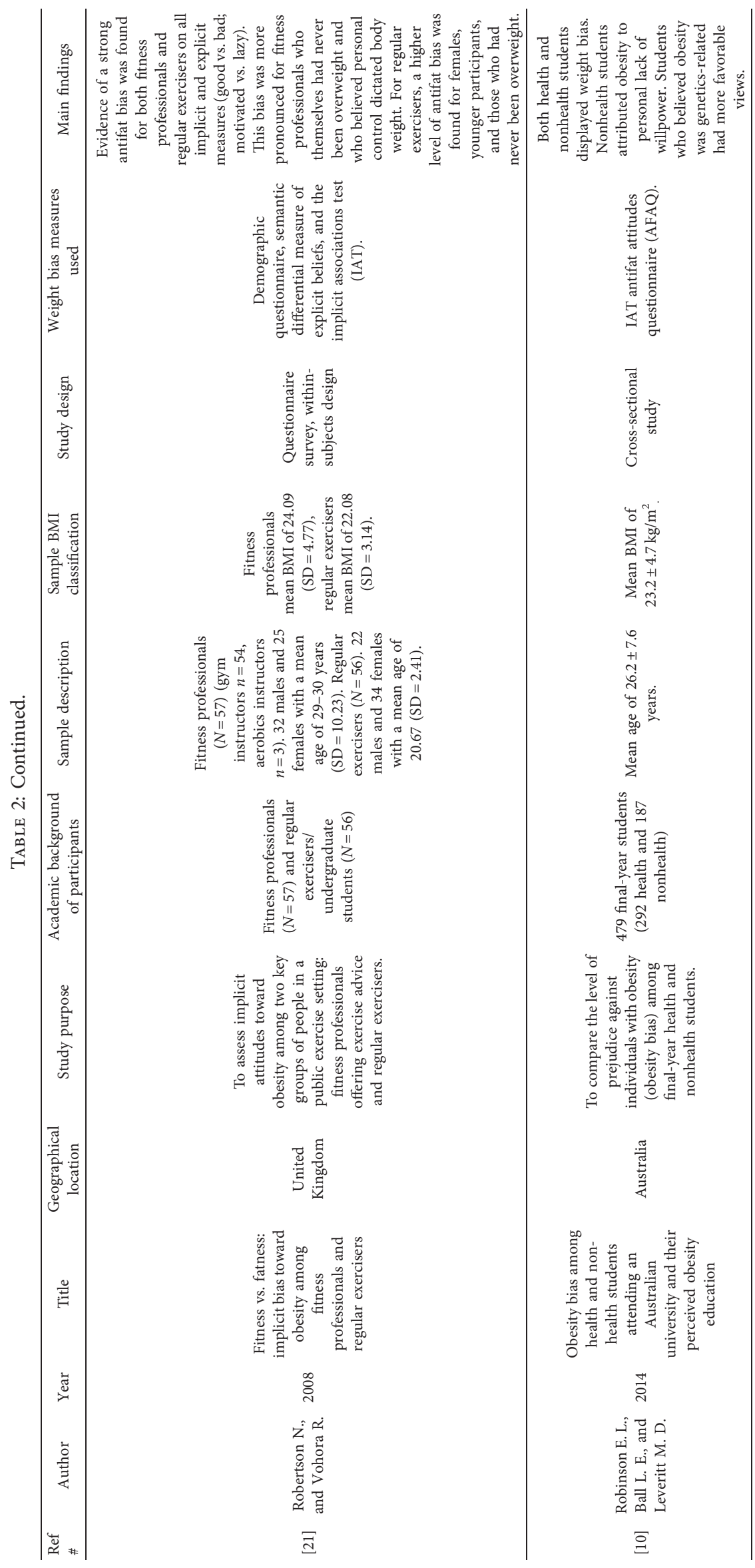




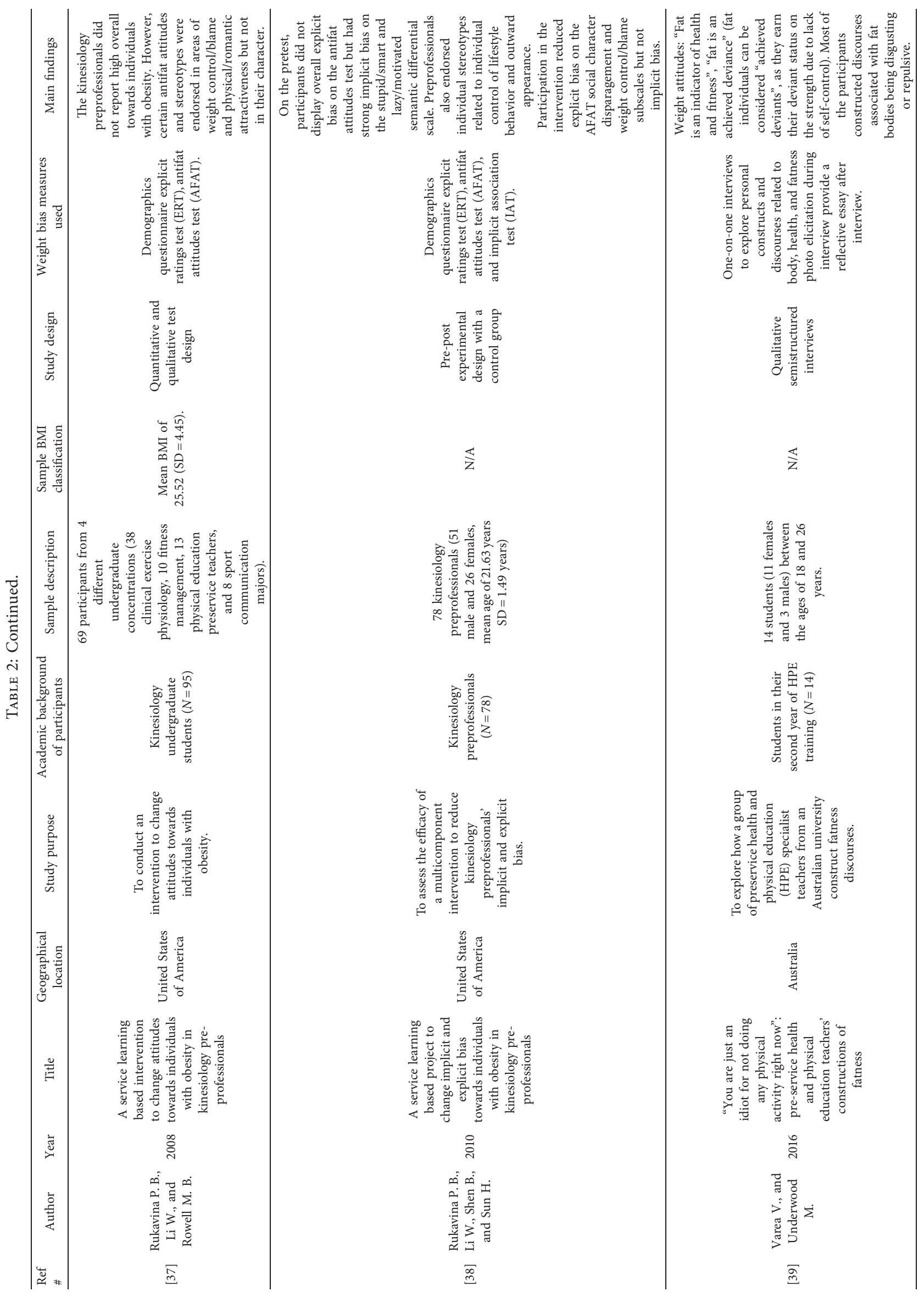




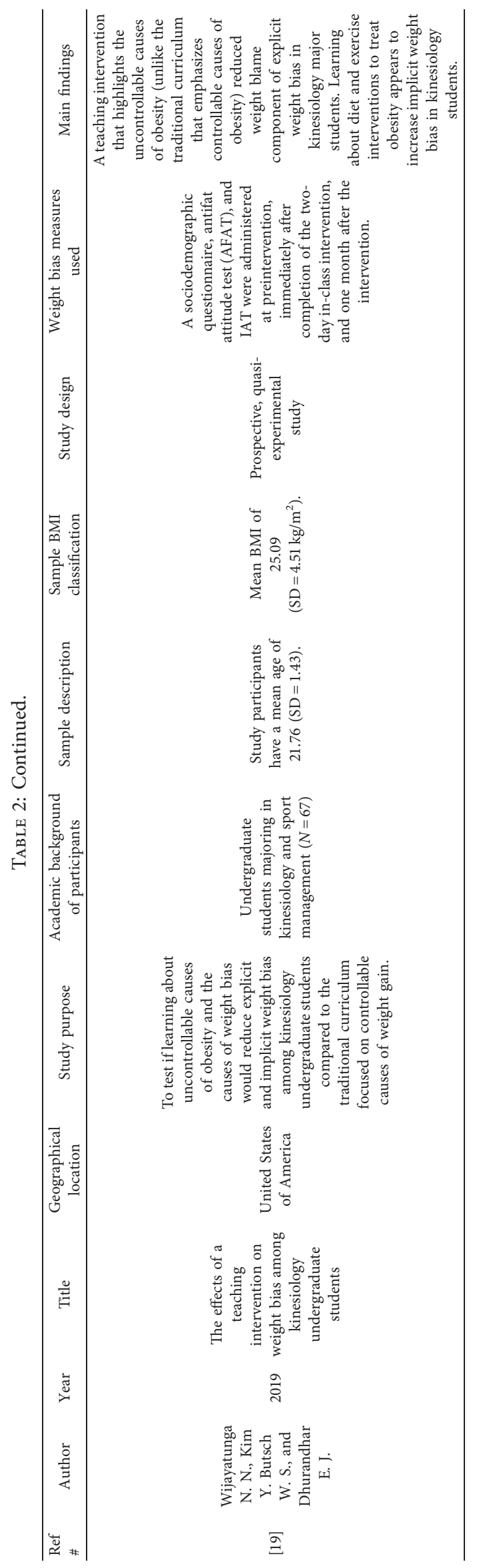


capabilities to foster student's learning and engagement" [35]. Results showed that physical education trainees with higher levels of perceived self-efficacy displayed more favorable attitudes toward the educational treatment of children and youth obesity [35]. Another study sampled professors of physical education majors who consistently believed that "physical education teachers should not be obese, since they are role models for their students" [31]. A similar perception was also found in an earlier study conducted by Hare et al., 2000, whereby a sample of health fitness instructors, exercise test technologists, and exercise specialists believed that they should maintain normal weight to be role models for their clients/patients [33]. According to this study, most of the information on weight control was derived from textbooks, college courses, and scientific data [33].

3.7. Knowledge of Obesity. Three studies measured exercise science students' and professionals' knowledge of obesity in relation to weight bias $[10,19,29]$. One study examined perceived obesity education and found that health students who poorly rated their knowledge regarding the genetic causes of obesity had higher explicit weight bias on the "blame" subscale [10]. Kinesiology students enrolled in a nontraditional curriculum intervention emphasizing uncontrollable causes of weight (i.e., genetics) decreased explicit weight bias on the "blame" subscale compared to the control group of students who were learning the traditional curriculum focused on the role of exercise and diet in weight management [19]. One out of the three studies did not find a significant association between health educators' knowledge of obesity and weight bias [29].

3.8. Lack of Personal History, Family, or Friend with Obesity. A lack of personal history of overweight predicted high implicit weight bias measures of "bad" and "lazy" among a sample of fitness professionals and regular exercisers [21]. Chambliss et al., 2004, showed that a lack of family history of obesity and a lack of friends with obesity were associated with higher explicit weight bias [18]. However, DeBarr and Pettit reported that there were no statistical differences in weight bias between health educators who were overweight compared to their normal weight peers [29].

\section{Discussion}

In this scoping review, 18 studies were reviewed to identify predictors of weight bias among exercise science students and professionals. In the following section, we identify gaps from each of the six themes, discuss future research directions, and outline the strengths and limitations of this scoping review.

4.1. Future Research and Recommendations. This scoping review identified studies in undergraduate students in physical education, exercise science, and kinesiology and two studies assessed professionals in fitness instruction health education. To our knowledge, we could not find studies that assessed predictors of weight bias among practicing kinesiologists, physiotherapists, and athletic therapists although one study assessed a mixed sample of exercise professionals including sports physiologists [30]. Future research is also warranted to examine predictors of weight bias in other health sectors and settings (e.g., public health). While studies have shown that weight bias from primary care providers negatively affects quality of care and healthcare utilization of patients with obesity [11], impacts on the behaviors, treatment, and quality of care of individuals with obesity have yet to be assessed systematically in exercise science and physical education practice settings.

Only three studies measured knowledge of obesity in relation to weight bias in exercise science students and professionals in this scoping review $[10,19,29]$. To better understand exercise science students and professionals' behaviors toward overweight and obesity, future research should seek to examine the contents of exercise science course curricula that may foster and potentially sustain weight bias in exercise science students. Because it has been shown that weight bias increases in physical education students as they progress through their educational programs [17], it should also be determined if weight bias increases from the start to the completion of exercise science programs as well. Future weight bias reduction interventions should be designed to address these potential predictors of weight bias and evaluate their impact throughout students' educational training in exercise science programs.

Eight of the studies included in this scoping review identified beliefs of controllability of weight as a predictor of weight bias [18, 21, 23, 33, 36-39]. Crandall (1994) coined the phrase "ideology of blame" to define the dominant social belief that individuals are personally responsible for their weight. This social belief may explain exercise science students and professionals' weight bias. Studies also show that exposure to simulated courses emphasizing the controllable aspect of weight and the rigid concepts of "eating less and moving more" may lead to higher weight bias [40]. One study showed that exercise science students believed obesity to be preventable and controllable through diet restriction and energy expenditure highly valuing diet and exercise for weight management and weight loss [39]. The study suggested a lack of knowledge on other therapeutic interventions including bariatric surgery and that there is still resistance on understanding obesity as a complex condition [39]. This paper highlights the need to increase awareness of the complexity of obesity in the curriculum offered to exercise science students and more research to understand the causes of students' resistance to adopt and learn new concepts about obesity.

Few studies exist to explain sex differences in weight bias attitudes and have shown mixed results [18, 21, 29, 34, 35]. It is unclear how sex or gender plays a role in weight bias [34]. Although it has been proposed that women may be more sensitive to weight bias due to their higher vulnerability to the "thin ideal" [21, 41], further research studies should be designed to be adequately powered to examine potential sex and gender differences and in exercise science students and 
professionals. It would also be important to determine causes of differences in weight bias between sexes and genders in this field.

Other areas that warrant further study are the potential influences of ethnicity and setting on the development of weight bias (i.e., how are individuals with obesity seen when observed in a neutral setting versus being seen in an exercise facility/setting). Two articles showed mixed results in ethnicity as a potential predictor of weight bias in exercise science students $[18,28]$; one stated no differences in explicit weight bias between American and Mexican athletes [28] while another found that exercise science students of Caucasian ethnicity living in a rural environment had higher levels of weight bias compared to those of other ethnicities [18]. With regard to setting, one study found that fitness center employees exhibited moderately strong implicit weight biases regardless of the setting in which they found themselves in (i.e., both neutral and exercise settings) [30]. The context in which the weight bias judgments were made did not affect the strength of implicit weight bias [30], suggesting weight bias still exists regardless of context in this aforementioned study. More research is needed to determine whether setting may act as a predictor of weight bias in fitness trainees and professionals.

One study by Fontana et al. evaluated the attitudes of professors in physical education departments toward individuals with obesity [31]. The sample of professors teaching physical education in this study held high implicit weight bias and disapproved physical education teachers with obesity as role models to students. This study demonstrated that greater explicit weight bias was associated with stronger disapproval of physical educators who have obesity as roles models and accepting physical education student majors living with obesity [31]. An earlier study by Hare et al. also showed that exercise professionals thought they should maintain normal weight to be role models for their clients [33]. These findings suggest a potential relationship between the importance exercise science students and professionals place on appearance and body weight (appearance orientation and body image preoccupation) with their current or future career as exercise science professionals. This suggests that more research is warranted on internalized weight bias ("the belief that negative stereotypes about weight apply to the self" [42]) in exercise science students and professionals to better understand underlying root causes of these internalized beliefs about weight, body image, and being role models in their field.

4.2. Strengths and Limitations. The present study is the first, to our knowledge, to gather the existing literature on predictors of weight bias in exercise science students and professionals. This scoping review provides a comprehensive summary of the overarching themes that emerged from the published studies that explored this topic. This comprehensive review helps identify predictors of weight bias that can serve as potential targets to address with curriculum changes and interventions aimed to improve training on the complexity of obesity and reduce weight bias in the early formative years before students become professionals in the field. However, since our scoping review focused on predictors of weight bias in exercise science students and professionals, suggestions for future research and interventions drawn from this paper can only be made about students and professionals in exercise science, kinesiology, and physical education fields.

\section{Conclusion}

This scoping review identified many overarching themes that predict weight bias in exercise science students and professionals. Belief in the personal controllability of weight was found to be the most researched and consistent predictor of weight bias in our population of interest. There appeared to be sex differences in weight bias that warrants further study; enrollment in a health sciences-related degree or program; psychosocial and personal factors relating to philosophy and personalities; traditional knowledge of obesity focusing mostly on diet and exercise; and lastly, a lack of personal history, family, or friend with obesity. Future research studies are needed to better understand predictors of weight bias in other health and exercise science-related fields, understand the impact of curricula that is heavily based on lifestyle factors only such as diet and exercise on weight bias, and evaluate the impact of weight bias reduction interventions in undergraduate students and professionals in the field of exercise science, kinesiology, and physical education.

\section{Appendix}

Database search strategy 2017 and 2019 is as follows:

(1) Anti-fat[tiab] OR "anti fat"[tiab] OR "fat phobia”[tiab] OR "fat phobic"[tiab]

(2) "Body Mass Index"[Mesh] OR "Body Weight"[Mesh] OR “obesity”[Mesh] OR “overweight”[Mesh] OR obese[tiab] OR obesity[tiab] OR overweight [tiab] OR “overweight"[tiab] OR weight[tiab] OR "Obesity/psychology”[Mesh]

(3) "Bias (Epidemiology)"[Mesh] OR "prejudice"[Mesh] OR "Social Stigma”[Mesh] OR "stereotyping” [MeSH] OR bias[tiab] OR biased[tiab] OR biases[tiab] OR discriminate[tiab] OR discriminates[tiab] OR discriminated[tiab] OR discrimination[tiab] OR prejudice[tiab] OR prejudiced[tiab] OR stereotype[tiab] OR stereotypes[tiab] OR stereotyped[tiab] OR stereotyping[tiab] OR stigma[tiab] OR stigmas [tiab] OR stigmatization[tiab] OR stigmatize[tiab] OR stigmatized[tiab] OR stigmatizes[tiab] OR stigmatizing[tiab] OR stigmatisation[tiab] OR stigmatise[tiab] OR stigmatised[tiab] OR stigmatises[tiab] OR stigmatising[tiab] OR empathy[tiab] OR trust[tiab] OR "Negative interaction" [tiab] OR "negative encounter"[tiab] OR "negative experience" [tiab] OR shame[tiab] OR shaming[tiab] OR shamed[tiab] OR “Attitude to Health"[Mesh] OR "Attitude of Health Personnel"[Mesh] OR "Health Knowledge, Attitudes, Practice"[Mesh] OR "Prejudice"[Mesh] 
(4) \#1 OR (\#2 AND \#3)

(5) "Physical Education and Training"[Mesh] OR "Health Occupations/education"[Mesh] OR "Students, Health Occupations/psychology"[Mesh]

(6) "Health science" [tiab] OR "health sciences" [tiab] OR "exercise science" [tiab] OR "exercise sciences"[tiab] OR "kinesiology"[tiab] OR "physiology" [tiab] OR "physical education"[tiab]

(7) "Pre-professionals" [tiab] OR "prehealth professional" [tiab] OR "non-specialist" [tiab] OR "trainee" [tiab ] OR "student" [tiab] OR "students" [tiab]

(8) \#5 OR (\#6 AND \#7)

(9) "Physical Fitness/psychology"[Mesh] OR "Fitness Centers/manpower" [Mesh]

(10) "Fitness"[tiab] OR "kinesiologists"[tiab] OR "kinesiologist"[tiab] OR "physical education"[tiab] OR "kinesiology"[tiab] OR "physiology" [tiab] OR "Health professional" [tiab] OR "Health professionals"[tiab]

(11) Specialist[tiab] OR trainer[tiab] OR employee[tiab] OR professional[tiab] OR professionals[tiab] OR specialists[tiab] OR employees[tiab] OR teacher [tiab] OR teachers[tiab] OR instructor[tiab] OR instructors[tiab]

(12) \#9 OR (\#10 AND \#11)

(13) \#4 AND (\#8 OR \#12)

Filters:

Date: 1990/01/01 to current (Search conducted December 14, 2017 and updated May 8, 2019)

Language: English OR French

Search conducted in Eric on EBSCOhost on December 14, 2017 and updated May 8, 2019.

(1) (weight OR fat OR anti-fat OR obesity OR obese OR Overweight) AND (bias OR stigma OR negative attitude OR Prejudice)

(2) (Educator OR Teacher OR Professional OR Personnel OR professors OR instructors OR students) AND (health OR fitness OR physical education OR kinesiology OR exercise science OR allied Health OR physiology)

(3) (Athletic therapist OR Physiologists OR kinesiologists OR athlete OR pre-service teacher)

(4) S1 AND (S2 OR S3)

\section{Data Availability}

The data supporting this scoping review are from previously reported studies and datasets, which have been cited. The processed data are available from the corresponding author upon request.

\section{Conflicts of Interest}

The authors declare no conflicts of interest regarding the publication of this article.

\section{Acknowledgments}

The authors are grateful for Ms. Katharine Hall, the librarian who assisted with database and keywords searches of the literature at Concordia University, Montreal. Dr. Alberga gratefully acknowledges Les Fonds de Recherche du QuébecSanté for her Chercheur Boursier Junior 1 Award (\#35277) held at Concordia University, Montreal.

\section{References}

[1] M. S. Rendall, M. M. Weden, C. Lau, P. Brownell, Z. Nazarov, and M. Fernandes, "Evaluation of bias in estimates of early childhood obesity from parent-reported heights and weights," American Journal of Public Health, vol. 104, no. 7, pp. 1255-1262, 2014.

[2] S. M. Phelan, D. J. Burgess, S. E. Burke et al., "Beliefs about the causes of obesity in a national sample of 4 th year medical students," Patient Education and Counseling, vol. 98, no. 11, pp. 1446-1449, 2015.

[3] S. Nutter, A. Ireland, A. S. Alberga et al., "Weight bias in educational settings: a systematic review," Current Obesity Reports, vol. 8, no. 2, pp. 185-200, 2019.

[4] M. Hebl and J. Xu, "Weighing the care: physicians' reactions to the size of a patient," International Journal of Obesity, vol. 25 , no. 8, pp. 1246-1252, 2001.

[5] G. A. Loomis, K. P. Connolly, C. R. Clinch, and D. A. Djuric, "Attitudes and practices of military family physicians regarding obesity," Military Medicine, vol. 166, no. 2, pp. 121-125, 2001.

[6] A. S. Alberga, L McLaren, S Russell-Mayhew, and K. M von Ranson, "Canadian senate report on obesity: focusing on individual behaviours versus social determinants of health may promote weight stigma," Journal of Obesity, vol. 2018, Article ID 8645694, 7 pages, 2018.

[7] X. Ramos Salas, "The ineffectiveness and unintended consequences of the public health war on obesity," $\mathrm{Ca}$ nadian Journal of Public Health, vol. 106, no. 2, pp. e79-81, 2015.

[8] X. R. Salas, M Forhan, T Caulfield, A. M Sharma, and K Raine, "A critical analysis of obesity prevention policies and strategies," Canadian journal of public health = Revue canadienne de sante publique, vol. 108, no. 5-6, pp. e598-e608, 2017.

[9] R. Puhl and K. D. Brownell, "Bias, discrimination, and obesity," Obesity Research, vol. 9, no. 12, pp. 788-805, 2001.

[10] E. L. Robinson, L. E. Ball, and M. D. Leveritt, "Obesity bias among health and non-health students attending an Australian university and their perceived obesity education," Journal of Nutrition Education and Behavior, vol. 46, no. 5, pp. 390-395, 2014.

[11] A. S. Alberga, I. Y. Edache, M. Forhan, and S. RussellMayhew, "Weight bias and health care utilization: a scoping review," Primary Health Care Research \& Development, vol. 20, p. e116, 2019.

[12] C. C. Irwin, C. W. Symons, and D. L. Kerr, "The dilemmas of obesity how can physical educators help?” Journal of Physical Education, Recreation \& Dance, vol. 74, no. 6, pp. 33-39, 2003.

[13] S. M. Phelan, D. J. Burgess, M. W. Yeazel, W. L. Hellerstedt, J. M. Griffin, and M. Ryn, "Impact of weight bias and stigma on quality of care and outcomes for patients with obesity," Obesity Reviews, vol. 16, no. 4, pp. 319-326, 2015.

[14] R. L. Pearl and R. M. Puhl, "The distinct effects of internalizing weight bias: an experimental study," Body Image, vol. 17, pp. 38-42, 2016. 
[15] M. Forhan and X. R. Salas, "Inequities in healthcare: a review of bias and discrimination in obesity treatment," Canadian Journal of Diabetes, vol. 37, no. 3, pp. 205-209, 2013.

[16] A. S. Alberga, B. J. Pickering, K. Alix Hayden et al., "Weight bias reduction in health professionals: a systematic review," Clinical Obesity, vol. 6, no. 3, pp. 175-188, 2016.

[17] K. S. O’Brien, J. A. Hunter, and M. Banks, "Implicit anti-fat bias in physical educators: physical attributes, ideology and socialization," International Journal of Obesity, vol. 31, no. 2, pp. 308-314, 2007.

[18] H. O. Chambliss, C. E. Finley, and S. N. Blair, "Attitudes toward obese individuals among exercise science students," Medicine \& Science in Sports \& Exercise, vol. 36, no. 3, pp. 468-474, 2004.

[19] N. N. Wijayatunga, Y. Kim, W. S. Butsch, and E. J. Dhurandhar, "The effects of a teaching intervention on weight bias among kinesiology undergraduate students," International Journal of Obesity, vol. 43, pp. 2273-2281, 2019.

[20] C. Greenleaf and K. Weiller, "Perceptions of youth obesity among physical educators," Social Psychology of Education, vol. 8, pp. 407-423, 2005.

[21] N. Robertson and R. Vohora, "Fitness vs. fatness: implicit bias towards obesity among fitness professionals and regular exercisers," Psychology of Sport and Exercise, vol. 9, no. 4, pp. 547-557, 2008.

[22] M. S. Faith, M. A Leone, T. S Ayers, M Heo, and A Pietrobelli, "Weight criticism during physical activity, coping skills, and reported physical activity in children," Pediatrics, vol. 110, no. 1, p. e23, 2002.

[23] M. Lynagh, K. Cliff, and P. J. Morgan, "Attitudes and beliefs of nonspecialist and specialist trainee health and physical education teachers toward obese children: evidence for "anti-fat" bias," Journal of School Health, vol. 85, no. 9, pp. 595-603, 2015.

[24] P. B. Rukavina and W. Li, "School physical activity interventions: do not forget about obesity bias," Obesity Reviews: An Official Journal of the International Association for the Study of Obesity, vol. 9, no. 1, pp. 67-75, 2008.

[25] H. Arksey and L. O'Malley, "Scoping studies: towards a methodological framework," International Journal of Social Research Methodology, vol. 8, no. 1, pp. 19-32, 2005.

[26] M. T. Pham, A. Rajić, J. D. Greig, J. M. Sargeant, A. Papadopoulos, and S. A. McEwen, "A scoping review of scoping reviews: advancing the approach and enhancing the consistency," Research Synthesis Methods, vol. 5, no. 4, pp. 371-385, 2014.

[27] A. C. Tricco, E. Lillie, W. Zarin et al., "PRISMA extension for scoping reviews (PRISMA-ScR): checklist and explanation," Annals of Internal Medicine, vol. 169, no. 7, pp. 467-473, 2018.

[28] M. Alameda and J. Whitehead, "Comparing levels of anti-fat bias between american and mexican athletes and undergraduate physical education and exercise science students," The Physical Educator, vol. 72, pp. 1-22, 2015.

[29] K. DeBarr and M. Pettit, "Weight matters: health educators' knowledge of obesity and attitudes toward people who are obese," American Journal of Health Education, vol. 47, no. 6, pp. 365-372, 2016.

[30] J. A. Dimmock, B. E. Hallett, and J. R. Grove, “Attitudes toward overweight individuals among fitness center employees: an examination of contextual effects," Research Quarterly for Exercise \& Sport, vol. 80, no. 3, pp. 641-647, 2009.

[31] F. Fontana, O. Furtado, O. Mazzardo, D. Hong, and W. de Campos, "Anti-fat bias by professors teaching physical education majors," European Physical Education Review, vol. 23, no. 1, pp. 127-138, 2017.

[32] C. Greenleaf, S. B. Martin, and D. Rhea, "Fighting fat: how do fat stereotypes influence beliefs about physical education?" Obesity, vol. 16, no. S2, 2008.

[33] S. W. Hare, J. H Price, M. G Flynn, and K. A King, "Attitudes and perceptions of fitness professionals regarding obesity," Journal of Community Health, vol. 25, no. 1, pp. 5-21, 2000.

[34] J. Langdon, P. Rukavina, and C. Greenleaf, "Predictors of obesity bias among exercise science students," Advances in Physiology Education, vol. 40, no. 2, pp. 157-164, 2016.

[35] E. Martínez-López, M. Zagalaz Sánchez, M. Ramos Álvarez, and M. de la Torre Cruz, "Self-efficacy expectations in teacher trainees and the perceived role of schools and their physical education department in the educational treatment of overweight students," European Physical Education Review, vol. 16, no. 3, pp. 251-266, 2010.

[36] L. A. Richardson, C. L. Fister, and S. E. Ramlo, "Effect of an exercise and weight control curriculum: views of obesity among exercise science students," Advances in Physiology Education, vol. 39, no. 2, pp. 43-48, 2015.

[37] P. B. Rukavina, W. Li, and M. B. Rowell, "A service learning based intervention to change attitudes toward obese individuals in kinesiology pre-professionals," Social Psychology of Education, vol. 11, no. 1, pp. 95-112, 2008.

[38] P. B. Rukavina, W. Li, B. Shen, and H. Sun, "A service learning based project to change implicit and explicit bias toward obese individuals in kinesiology pre-professionals," Obesity Facts, vol. 3, no. 2, pp. 117-126, 2010.

[39] V. Varea and M. Underwood, "You are just an idiot for not doing any physical activity right now," European Physical Education Review, vol. 22, no. 4, pp. 465-478, 2016.

[40] B. A. Teachman, K. D. Gapinski, K. D. Brownell, M. Rawlins, and S. Jeyaram, "Demonstrations of implicit anti-fat bias: the impact of providing causal information and evoking empathy," Health Psychology, vol. 22, no. 1, pp. 68-78, 2003.

[41] P. A. Klaczynski, K. W. Goold, and J. J. Mudry, "Culture, obesity stereotypes, self-esteem, and the "thin ideal": a social identity perspective," Journal of Youth and Adolescence, vol. 33, no. 4, pp. 307-317, 2004.

[42] R. D. Marshall, J. D. Latner, and A. Masuda, "Internalized weight bias and disordered eating: the mediating role of body image avoidance and drive for thinness," Frontiers in Psychology, vol. 10, p. 2999, 2019. 